\title{
WS3-4
}

\section{Epigenetics of cell adhesion genes in human prostate cancer}

Shimane University ${ }^{1)}$, Kagoshima University ${ }^{2)}$, UCSF and VA Medical Center ${ }^{3)}$

Hiroaki Shiina ${ }^{1}$, Hideki Enokida ${ }^{2)}$, Masayuki Nakagawa ${ }^{2)}$, Christopher J Kane ${ }^{3)}$, Rajvir Dahiya ${ }^{3)}$, Mikio Igawa"

Cell adhesion molecules such as E-cadherin and catenins, are directly interrelated with each other to maintain epithelial cell polarity. Functional loss of these molecules may be involved in initiation, progression and metastasis of various cancers. Mechanisms of inactivation of cell adhesion genes may be through $\mathrm{CpG}$ methylation and mutation. We hypothesized that promoter $\mathrm{CpG}$ hypermethylation of cell adhesion genes are involved in the inactivation of these genes and trigger initiation, progression and metastasis of prostate cancer. To test this hypothesis, prostate cancer samples were analyzed for gene expression and $\mathrm{CpG}$ methylation of E-cadherin, beta-catenin, gamma-catenin and $\mathrm{CD} 44$ genes.

All Japanese samples (191 radical prostatectomies and 96 pathologically proven BPH) were obtained at Shimane University Hospital between 1999 June and 2003 May. CpG methylation was evaluated by methylation specific PCR (MSP) and bisulfite DNA sequencing. The primers to detect $\mathrm{CpG}$ methylation of E-cadherin, beta-catenin and CD44 include putative SP1 sites. For gamma-catenin, two different sets of MSP primers (A ; distal $\mathrm{CpG}$ sites and $\mathrm{B}$ : proximal $\mathrm{CpG}$ sites) were used. Gene expression was evaluated by RT-PCR.

The frequencies of methylation in prostate cancer (PC) were $39 \%$ for E-cadherin, $96 \%$ for gamma-catenin (A), 40\% for gamma-catenin (B), 37\% for beta-catenin and 49\% for $\mathrm{CD} 44$, whereas those in $\mathrm{BPH}$ were $4 \%$ for E-cadherin, $93 \%$ for gamma-catenin (A), 37\% for gamma-catenin (B), $1 \%$ for beta-catenin and 7\% for CD44. Down-regulation of mRNA transcripts was observed in cancers with $\mathrm{CpG}$ hypermethylation of E-cadherin, betacatenin, gamma-catenin (B) and CD44, but not of gamma-catenin (A). Among these genes, only CD44 methylation was related with higher Gleason score. On the other hand, beta-catenin methylation was significantly correlated with higher preoperative PSA value and capsular extension.

These findings suggested that epigenetic alterations in cell-adhesion molecule genes are involved in the pathogenesis of prostate cancer progression.

\section{WS3-5 \\ Elevated expression of Valosin-containing protein (p97) is associated with poor progno-
sis of prostate cancer}

Departments of Pathology Osaka University Graduate School of Medicine, Osaka, Japan ${ }^{12}$, Surgery and Clinical Oncology Osaka University Graduate School of Medicine, Osaka, Japan ${ }^{2)}$, Urology Osaka University Graduate School of Medicine, Osaka, Japan ${ }^{3)}$, Division of Urology Osaka National Hospital, Osaka, Japan ${ }^{4)}$ Yuichi Tsujimoto $^{1)}$, Yasuhiko Tomita ${ }^{1)}$, Yoshihiko Hoshida ${ }^{1)}$, Takuro Kono', Toshitsugu Oka ${ }^{4)}$, Shinji Yamamoto ${ }^{3)}$, Norio Nonomura ${ }^{3)}$, Akihiko Okuyama ${ }^{3)}$, Katsuyuki Aozasa ${ }^{1\rangle}$

Valosin-containing protein (VCP) was shown to be associated with metastasis and prognosis in human cancers. In the present study, association of VCP expression with recurrence and prognosis of patients with PCA receiving conservative therapies was examined immunohistochemically in 136 patients. Staining intensity of tumor cells was categorized as weaker (level 1) or equal to or stronger (level 2) than that in endothelial cells. $23.5 \%$ of patients showed level 1, and 76.5\%level 2 VCP expression. Quantitative RT-PCR analysis revealed higher VCP mRNA expression in level $2(n=5)$ than level 1 patient cases $(n=5)(p<0.05)$. Significant difference was observed between VCP level 1 and 2 patients in positive rate of digital rectal examination, serum PSA level, cancer volume, Gleason score, stage, progression-free and overall survival (for both). Multivariate analysis revealed VCP expression level, serum PSA level, and Gleason score to be independent prognosticators for progression-free and overall survivals. Progression of PCA was found in $9.4 \%$ of level 1 but in $64 \%$ of level 2 patients $(\mathrm{p}<0.0001)$. PCA with level 1 VCP expression could be treated conservatively. 